\title{
A New Wall Shear Stress Model for Atmospheric Boundary Layer Simulations
}

\author{
MARCUS HULTMARK \\ Department of Mechanical and Aerospace Engineering, Princeton University, Princeton, New Jersey \\ MARC CALAF* AND Marc B. Parlange \\ School of Architecture, Civil and Environmental Engineering, École Polytechnique Fédérale de Lausanne, Lausanne, Switzerland
}

(Manuscript received 20 September 2012, in final form 27 June 2013)

\begin{abstract}
A new wall shear stress model to be used as a wall boundary condition for large-eddy simulations of the atmospheric boundary layer is proposed. The new model computes the wall shear stress and the vertical derivatives of the streamwise velocity component by means of a modified, instantaneous, and local law-of-thewall formulation. By formulating a correction for the modeled shear stress, using experimental findings of a logarithmic region in the streamwise turbulent fluctuations, the need for a filter is eliminated. This allows one to model the wall shear stress locally, and at the same time accurately recover the correct average value. The proposed model has been applied to both unique high Reynolds number experimental data and a suite of large-eddy simulations, and compared to previous models. It is shown that the proposed model performs equally well or better than the previous filtered models. A nonfiltered model, such as the one proposed, is an essential first step in developing a universal wall shear stress model that can be used for flow over heterogeneous surfaces, studies of diurnal cycles, or analyses of flow over complex terrain.
\end{abstract}

\section{Introduction}

One of the most promising tools for numerically simulating high Reynolds number turbulent flows, such as the flow in the atmospheric boundary layer (ABL), is large-eddy simulation (LES). In LES only the large turbulent eddies are resolved and numerically solved for, according to the governing equations. The small eddies (subgrid scales) are modeled using a subgrid-scale model. This method has revolutionized simulations of turbulent flows, capturing much of the physics and allowing for investigations of high Reynolds number flows. However, there are still limitations with LES. For example, introducing solid objects into the flow, such as mountains and forest canopies (Schumann 1990; Gong et al. 1996; Belcher et al. 2012; Shaw and Schumann 1992), or

\footnotetext{
* Current affiliation: Department of Mechanical Engineering, University of Utah, Salt Lake City, Utah.

Corresponding author address: Marcus Hultmark, Department of Mechanical and Aerospace Engineering, Princeton University, D222 Engineering Quadrangle, Olden Street, Princeton, NJ 08544. E-mail: hultmark@princeton.edu
}

defining time-/space-varying surfaces (Albertson and Parlange 1999a; Avissar et al. 1998; Bou-Zeid et al. 2004), creates problems close to the surface itself because of the complicated boundary conditions needed. Much recent work has focused on subgrid-scale models given the importance in extracting energy correctly from the resolved scales (e.g., Porté-Agel et al. 2001). A critical component for a high-quality LES of the ABL is the wall boundary condition, that is, the boundary condition at the grid point closest to the wall (the plane, including all "first" grid points, will be referred to as the wall layer). This is due to the intrinsic definition of the ABL being the region of the atmosphere in close contact with the ground surface and where most of the momentum and scalar exchanges take place. Therefore, it is crucial to properly capture these interactions in order to accurately model the ABL. It is common practice in LES of atmospheric flows to use a vertically staggered grid. Thus, the terms that need to be defined at the wall layer are the velocity gradients $d \tilde{u} / d z$ and $d \tilde{v} / d z$ as well as the wall shear stress $\tau_{w}$ (Moeng 1984), where $u$ and $v$ are the instantaneous velocity components in the plane parallel to the surface; the tilde implies a numerically resolved value (filtered by the numerical grid); and $z$ is 
the distance from the wall in the direction normal to the wall.

The most common approach for defining the velocity gradient at the wall layer is to relate it to the universal law of the wall (Prandtl 1925, 1932; Millikan 1939), here written following Tennekes and Lumley's (1972) notation:

$$
\frac{\left\langle u_{s}\right\rangle}{\sqrt{\left\langle\tau_{w}\right\rangle}}=\frac{1}{\kappa} \log \left(z^{+}\right)+B,
$$

where $\tau_{w}=\tau / \rho$ is the kinematic stress at the wall, $\tau$ is the shear stress exerted on the wall by the fluid, $\rho$ is the fluid density, and $z^{+}=z \sqrt{\left\langle\tau_{w}\right\rangle} / \nu$, where $v$ is the kinematic viscosity. The friction velocity $u_{*}$ is commonly used instead of the kinematic stress, where $u_{*}=\sqrt{\tau_{w}} ; u_{s}$ is the velocity in the direction of the mean velocity (streamwise direction), $\kappa=0.4$ is the von Kármán constant, and angle brackets indicate an average quantity. The logarithmic behavior of the mean streamwise velocity, as described above, can be expected to be found in high Reynolds number turbulent boundary layers with smooth surfaces, in the region where $z / \delta<0.12$ and $z^{+}>800$ (Hultmark et al. 2012) ( $\delta$ is the height of the shear layer). However, here we are mainly concerned with the atmospheric boundary layer where the surface is far from smooth. For rough surfaces with a near-neutral atmospheric stability (with a characteristic roughness length $z_{0}$ ) the log law can be written as (Monin and Obukhov 1954; Landau and Lifshitz 1959)

$$
\frac{\left\langle u_{s}\right\rangle}{\sqrt{\left\langle\tau_{w}\right\rangle}}=\frac{1}{\kappa} \log \left(\frac{z}{z_{0}}\right) .
$$

If $\left\langle u_{s}\right\rangle, z$, and $z_{0}$ are known, then one can solve for $\left\langle\tau_{w}\right\rangle$ as

$$
\left\langle\tau_{w}\right\rangle=\left[\frac{\kappa}{\log \left(z / z_{0}\right)}\right]^{2}\left\langle u_{s}\right\rangle^{2}=f\left(z / z_{0}\right)\left\langle u_{s}\right\rangle^{2} .
$$

The vertical gradient of the mean streamwise velocity can be written as follows:

$$
\frac{d\left\langle u_{s}\right\rangle}{d z}=\frac{\sqrt{\left\langle\tau_{w}\right\rangle}}{\kappa z}=\frac{\left\langle u_{s}\right\rangle \sqrt{f\left(z / z_{0}\right)}}{k z} .
$$

Unfortunately, this method requires knowledge about the magnitude and direction of the average velocity and it provides the average velocity gradient. In LES, only the resolved velocity $\tilde{u}$ in the wall layer is known, and what is required as an input is the gradient of the resolved velocity, not the average. In the literature there are several methods to account for the difference between the resolved velocity and its average. One can simply ignore the fact that these log laws are derived for average quantities and use them as if they were also valid instantaneously [as described in, e.g., Albertson and Parlange (1999b), this method will be referred to as the instantaneous logarithm (IL) method]. However, the nonlinearity in the log law causes this method to overpredict the average shear by a factor of $\left\langle\tilde{u}_{s}^{\prime 2}\right\rangle$ :

$$
\left\langle\tau_{w}\right\rangle^{\mathrm{IL}}=\left[\frac{\kappa}{\log \left(z / z_{0}\right)}\right]^{2}\left\langle\tilde{u}_{s}^{2}\right\rangle=\left[\frac{\kappa}{\log \left(z / z_{0}\right)}\right]^{2}\left(\left\langle\tilde{u}_{s}\right\rangle^{2}+\left\langle\tilde{u}_{s}^{\prime 2}\right\rangle\right),
$$

where $\tilde{u}_{s}^{\prime}$ is the fluctuating component of the resolved velocity, such as $\tilde{u}_{s}=\left\langle\tilde{u}_{s}\right\rangle+\tilde{u}_{s}^{\prime}$. The IL model is probably the most commonly used wall shear stress model in the community, even though its overprediction of the average shear stress is well known. One common method to account for the fluctuations of the resolved velocity was developed by Schumann (1975) and later improved by Grötzbach (1987) (hereafter the SG model); it approximates the deviation from the average wall shear stress as a linear function of the instantaneously resolved streamwise velocity component,

$$
\tilde{\tau}_{w}(x, y, z, t)^{\mathrm{SG}}=\left\langle\tau_{w}\right\rangle\left[\frac{\tilde{u}_{s}(x, y, z, t)}{\left\langle u_{s}\right\rangle}\right],
$$

where the mean wall shear is found by using Eq. (2) and the averaged velocity is approximated as the velocity averaged across the wall layer. The SG model is simple to implement numerically, but it has a weak physical foundation regarding the deviation from the mean shear stress. Piomelli et al. (1989) noted that the SG model could be improved by introducing a displacement to the evaluation point of the wall shear stress, such that

$$
\tilde{\tau}_{w}(x, y, z, t)^{\mathrm{SG}, \text { shifted }}=\left\langle\tau_{w}\right\rangle\left[\frac{\tilde{u}_{s}\left(x+\Delta_{s}, y, z, t\right)}{\left\langle u_{s}\right\rangle}\right],
$$

where $\Delta_{s}=z \cos (\alpha)$, corresponding to the inclination angle of large coherent structures at the wall. For high Reynolds number flows such as the ABL we are concerned within this study, $\alpha \approx 13^{\circ}-15^{\circ}$ (Stoll and Porté-Agel 2006; Marusic and Heuer 2007). Based on the same concept, of inclined large-scale coherent structures and corresponding sweep-eject events toward and away from the wall, Piomelli et al. (1989) introduced a new model based on the wall-normal direction of the velocity $w$. They argued that a sweep event produces positive shear stress fluctuation and an ejection event decreases the shear stress; therefore, they hypothesized that the wall-normal component of the velocity was better than 
the streamwise component to account for the effect of these events on the wall stress, such that

$\tilde{\tau}_{w}(x, y, z, t)^{\text {eject }}=\left\langle\tau_{w}\right\rangle-C\left\langle\tau_{w}\right\rangle^{1 / 2} \tilde{w}\left(x+\Delta_{s}, y, z, t\right)$,

where $C$ is a nondimensional constant of order 1 . Although this model has a stronger physical foundation than the SG model, its performance was worse in the comparison conducted by Marusic et al. (2001), who used experimental data to evaluate different models. In the same study Marusic et al. introduced a new model [hereafter the Marusic-Kunkel-Porté-Agel (MKP) model] based on the results from wind-tunnel experiments, where correlations between the velocity and the wall shear stress were measured. The MKP model is based on the same principle as the ejection model, but instead of using the wall-normal component of the velocity as an indicator for the sweep-eject events, it uses the streamwise component, such that

$$
\begin{aligned}
\tilde{\tau}_{w}(x, y, z, t)^{\mathrm{MKP}}= & \left\langle\tau_{w}\right\rangle-a\left\langle\tau_{w}\right\rangle^{1 / 2}\left[\tilde{u}_{s}\left(x+\Delta_{s}, y, z, t\right)\right. \\
& \left.-\left\langle\tilde{u}_{s}\left(x+\Delta_{s}, y, z, t\right)\right\rangle\right] .
\end{aligned}
$$

A common problem for the above-described models is that they require homogenous surfaces, since in order to find $\left\langle\tilde{u}_{s}\right\rangle$ one needs to average across the complete wall layer. A nonfiltered model is essential for simulations where the shear stress varies in space and time, such as flow over heterogeneous surfaces, the study of diurnal cycles, or the analysis of flow over complex terrain. BouZeid et al. (2005) introduced a model in which the velocity $\left[\left\langle\tilde{u}_{s}(x, y, z, t)\right\rangle\right]$ is only filtered over the adjacent grid points ( $2 \Delta$ filter), which will be referred to as the $2 \Delta$ model. This model, although still filtered, does not require the surface to be entirely homogeneous, which is a crucial step to make LES a viable option to simulate realistic atmospheric flows (Belcher et al. 2012; BouZeid et al. 2004, 2007; Hobson et al. 1999). However, similar to the IL model, the $2 \Delta$ model will overestimate the average shear stress [see Eq. (5)]. Nonetheless, BouZeid et al. showed that the $2 \Delta$ filter reduced the error by approximately $50 \%$ compared to the IL method.

The SG model, ejection model, and MKP model all have in common that the average wall shear stress will be correct (since it is an input to the models themselves). However, they also have in common that the modeled shear stress scales as $u_{s}$, where it is expected to scale as $u_{s}^{2}$ at these Reynolds numbers. This will affect the fluctuating part of the shear stress, which will affect higherorder moments and the spectrum. Also, the higher-order statistics of the $2 \Delta$ model are questionable, since it is based on filtered data. Overall, the MKP model should have an advantage over the other models, regarding the fluctuations, since it was developed with the frequency spectrum in mind; thus, at least the second-order statistics can be expected to behave more physically.

In this study we will compare the performance of several different models and their method for accounting for the fluctuations in the wall shear stress. We then present a new model based on the recent findings by Hultmark et al. (2012), who showed that the scaling of the streamwise turbulent fluctuations, in hydraulically smooth conditions, closely follow that of the mean velocity. In the region where the mean velocity exhibits a logarithmic behavior, the fluctuations do as well. They showed that the variance can be described as

$$
\frac{\left\langle u_{s}^{\prime 2}\right\rangle}{\left\langle\tau_{w}\right\rangle}=B_{1}-A_{1} \log \left(\frac{z}{\delta}\right)
$$

where the constants were found to be $B_{1}=1.61$ and $A_{1}=1.25$. Hultmark et al. (2013) extended these results to also include hydraulically rough flows, which are of great importance in the atmospheric boundary layer. As will be shown in this paper, this log law for the turbulent fluctuations opens the door for a wall boundary condition based on local quantities while still recovering the correct average shear. Using these findings a new mathematically consistent local model, which does not overpredict the average shear stress, will be introduced. The local formulation of this method makes it suitable to form the basis for LES over heterogeneous surfaces. It should, however, be pointed out that the proposed model as well as the other models considered in this study is based on the existence of the logarithmic law and the assumption that the shear stress can be modeled using the velocity field. This assumption might fail in the study of strongly heterogeneous surfaces and limit the usefulness of the models. Nevertheless, it will be shown that the proposed model performs as well or better than existing models, without the need for filtering.

\section{Proposed wall shear stress model}

The duality between the mean velocity profile and the variance profile allows one to use the log law for the turbulent fluctuations [Eq. (10)], as described by Hultmark et al. (2012), to find the wall shear stress similarly to the above-described methods, such that

$$
\left\langle\tau_{w}\right\rangle=\left[\frac{1}{A-B \log (z / \delta)}\right]\left\langle u_{s}^{\prime 2}\right\rangle=g(z / \delta)\left\langle u_{s}^{\prime 2}\right\rangle
$$

If this expression is used in a truly instantaneous manner (similar to the IL model), then the average wall shear 
stress will be overpredicted (with an amount related to the mean velocity). However, if the two log laws [Eqs. (3) and (11)], which are valid in the same region in space, are used simultaneously, then we can compensate for the overprediction of the IL model by relating the variance to the mean velocity as

$$
\left\langle u_{s}^{\prime 2}\right\rangle=\frac{f\left(z / z_{0}\right)}{g(z / \delta)}\left\langle u_{s}\right\rangle^{2}
$$

However, Eq. (12) is formulated for the variance of the instantaneous velocity, not the resolved velocity as is known in the LES. Smits et al. (2011) introduced a method to correct experimental data, acquired with poor spatial resolution in wall-bounded turbulent flows, by relating it to the attached eddy hypothesis by Townsend (1976). They were able to show that the filtered energy is inversely proportional to the wall-normal distance from the wall. Here, we will adopt the same method to describe the difference between the variance of the instantaneous and resolved velocity, assuming that the sensor length $\ell$ used by Smits et al. (2011) can be replaced by $\Delta$ [the cube root of the grid volume, $\left.\Delta=(d x d y d z)^{1 / 3}\right]$. Given that the ABL experiences very high Reynolds numbers, the relationship reduces to

$$
\frac{\left\langle\tilde{u}_{s}^{2}\right\rangle}{\left\langle u_{s}^{\prime 2}\right\rangle}=\frac{1}{1+0.1365(\Delta / z)} .
$$

Thus, by substituting Eq. (13) into Eq. (12), the IL model [Eq. (5)] can be rewritten as

$$
\left\langle\tau_{w}\right\rangle^{\mathrm{IL}}=f\left(z / z_{0}\right)\left[\left\langle\tilde{u}_{s}\right\rangle^{2}+\frac{1}{1+0.1365(\Delta / z)} \frac{f\left(z / z_{0}\right)}{g(z / \delta)}\left\langle u_{s}\right\rangle^{2}\right],
$$

and, since $\left\langle u_{s}\right\rangle=\left\langle\tilde{u}_{s}\right\rangle$, Eq. (14) can be further rearranged as

$$
\left\langle\tau_{w}\right\rangle^{\mathrm{IL}}=f\left(z / z_{0}\right)\left\langle\tilde{u}_{s}\right\rangle^{2}\left[1+\frac{1}{1+0.1365(\Delta / z)} \frac{f\left(z / z_{0}\right)}{g(z / \delta)}\right] .
$$

Thus, by comparing Eqs. (15) and (3), the overprediction caused by the nonlinearity can be predicted by knowing $f\left(z / z_{0}\right), g(z / \delta)$, and $\Delta / z$. We are interested in boundary conditions being implemented on a certain grid point, which has a known $z, z_{0}$, and $\delta$, which reduces $f\left(z / z_{0}\right)$ and $g(z / \delta)$ to constants at the first grid point (hereafter $f$ and $g$ ). The overprediction can now be found, and an entirely local method that will return the correct average value as well as the correct variance can be formulated as

$$
\tilde{\tau}_{w}^{\text {model }}=\frac{f}{1+\frac{1}{1+0.1365(\Delta / z)} \frac{f}{g}} \tilde{u}_{s}^{2} .
$$

The proposed model is based purely on instantaneous values, which will allow the shear stress to have realistic fluctuations that scale with $u_{s}^{2}$, which one would expect to be the correct scaling at high Reynolds numbers.

To incorporate this into a large-eddy simulation, knowledge about the direction of $u_{s}$ is needed, since it is not obvious that it aligns with the numerical grid. This exercise is needed for all of the above-mentioned models, since the log law is strictly defined in the streamwise direction. However, this poses a problem since the average direction is not known a priori. Here we propose to use the direction of the $2 \Delta$-filtered velocity (Bou-Zeid et al. 2005), such that

$$
\tilde{\tau}_{w, i}^{\text {model }}=\tilde{\tau}_{w}^{\text {model }} n_{i},
$$

where $i$ is the direction of interest in the plane parallel to the surface (1 or 2$)$ and

$$
n_{i}=\frac{\left\langle u_{i}\right\rangle_{2 \Delta}}{\sqrt{\left\langle u_{1}\right\rangle_{2 \Delta}^{2}+\left\langle u_{2}\right\rangle_{2 \Delta}^{2}}} .
$$

In an equivalent manner, the formulation for the vertical derivatives of the horizontal flow components can be written as

$$
\frac{\partial \tilde{u}_{i}}{\partial z}=\frac{\sqrt{\tilde{\tau}_{w}^{\text {model }}}}{\kappa z} n_{i} .
$$

\section{Results and discussion}

To evaluate the proposed model, its performance is compared to that of the SG model and the MKP model, both experimentally and numerically.

\section{a. Experimental data}

The data used for evaluation of the wall shear stress models were acquired in the Princeton/Office of Naval Research (ONR) Superpipe at an unprecedented Reynolds number [for more details about the experiments, see Hultmark et al. (2012); only the fifth case was used in this analysis, $\left.\operatorname{Re}_{\tau}=\delta \sqrt{\left\langle\tau_{w}\right\rangle} / \nu=2 \times 10^{4}\right]$. By using nanoscale thermal anemometer probes (NSTAPs) (Bailey et al. 2010; Vallikivi et al. 2011), measurements of the instantaneous velocity in the streamwise direction were obtained. For each wall-normal position, $2.7 \times 10^{7}$ samples were acquired with a sample rate of $300 \mathrm{kHz}$, after first 
TABLE 1. Comparison of resulting statistics from different models.

\begin{tabular}{lcc}
\hline \hline \multicolumn{1}{c}{ Source } & $\left\langle\tau_{w}\right\rangle\left(\mathrm{m}^{2} \mathrm{~s}^{-2}\right)$ & $\left\langle\tau_{w}^{\prime 2}\right\rangle\left(\mathrm{m}^{4} \mathrm{~s}^{-4}\right)$ \\
\hline At $y^{+}=4.38$ & 0.1566 & 0.0035 \\
Proposed model & 0.1585 & 0.0010 \\
SG model & 0.1583 & 0.0002 \\
MKP model & 0.1583 & 0.0013 \\
\hline
\end{tabular}

being low-pass filtered at $150 \mathrm{kHz}$. The extremely small size of the sensor and a bandwidth exceeding $300 \mathrm{kHz}$ allowed for unfiltered data acquisition, which is crucial for high accuracy, especially close to the wall (Smits et al. 2011). In these experiments the NSTAP was traversed away from the wall and instantaneous velocity data were recorded at several wall-normal positions, with the closest being only $14 \mu \mathrm{m}$ from the wall. A slightly elevated hotwire has been shown to be a good method to measure instantaneous wall shear stress (Chew et al. 1998; Khoo et al. 1998; Alfredsson et al. 1988). They showed that if the probe is positioned such that $z^{+}<5$, then the velocity profile can be expected to exhibit a linear profile such that $u / \sqrt{\tau_{w}}=z^{+}$. This allows for acquisition of the instantaneous wall shear stress, since both the velocity and the kinematic viscosity are known. The first wall-normal point in this study (14 $\mu \mathrm{m}$ from the wall) corresponds to $z^{+}=z \sqrt{\left\langle\tau_{w}\right\rangle} / \nu=4.38$ for the studied Reynolds number. Thus, the velocity data from this location can be used to estimate the instantaneous wall shear stress.

The SG model, MKP model, and the proposed model can all be applied to the instantaneous streamwise velocity measured within the logarithmic region, and the modeled shear stress $\tau_{w, m}$ can be compared to the measured actual wall shear stress. The models were applied to data acquired at $z / \delta=0.0516$, which corresponds to $z^{+}=$ 1048 , which is within the logarithmic region as reported by Hultmark et al. (2012). At this position the sample length corresponds to approximately $2.2 \times 10^{4}$ integral length scales, which ensures well-converged statistics. The resulting averages and variances of the shear stresses are summarized in Table 1.

It is clear that all methods capture the average shear stress fairly well, but this is not surprising for the MKP model and the SG model, since they require it as an input. The resulting average shear stress from the proposed model matched that of the MKP model and the SG model within $0.2 \%$ without requiring it as an input, indicating that the correction based on the variance introduced in this study performs well. It is interesting to note that all models underestimate the variance of the

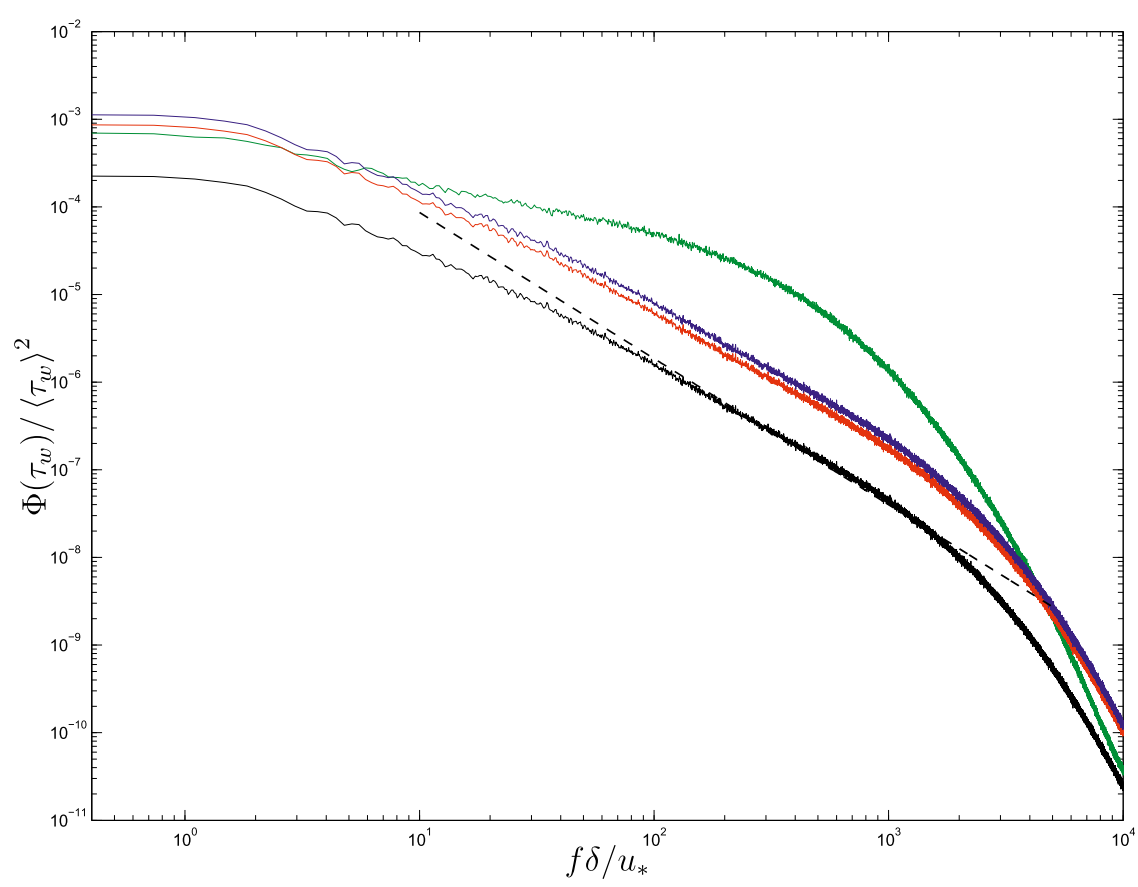

FIG. 1. Frequency spectrum of measured and modeled wall shear stresses. It is clear that the MKP model (blue line) and the proposed model (red line) are more similar to the experimentally measured data (green line) compared to the SG model (solid black line). All the models differ substantially in shape compared to the measured data, where the intermediate frequencies are underpredicted. The dashed black line represents the $\left(f \delta / u_{\tau}\right)^{-5 / 3}$, corresponding to the inertial subrange. 

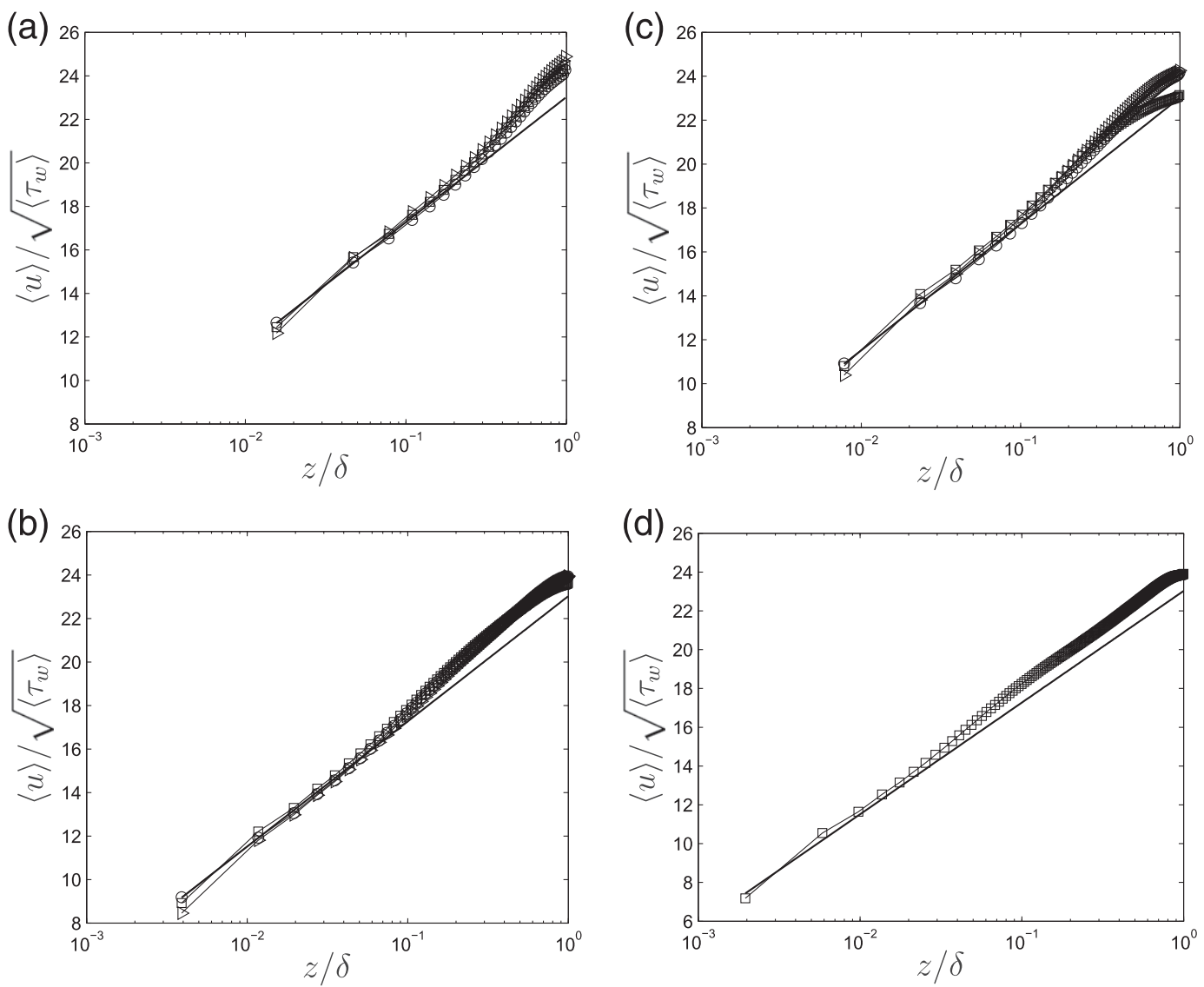

FIG. 2. Vertical profiles of the mean streamwise velocity normalized by $\sqrt{\left\langle\tau_{w}\right\rangle}$. The solid black line represents the expected logarithmic profile, $\langle\tilde{u}\rangle / \sqrt{\left\langle\tau_{w}\right\rangle}=1 / \kappa \log \left(z / z_{0}\right)$, while the open circles, squares, and triangles represent the SG model, the proposed model, and the MKP model, respectively. The numerical resolutions (a) $32^{3}$, (b) $64^{3}$, (c) $128^{3}$, and (d) $256^{3}$ are presented.

wall shear stress, especially the SG model, which is more than an order of magnitude smaller. The MKP model and the proposed model are better, but still much too low. To understand the underestimation, the frequency spectrum of the modeled shear stress is plotted in Fig. 1. Here it is obvious that the shape of all three models differs quite substantially from that of the measured wall shear stress. This indicates that using the velocity in the logarithmic layer might not be the ideal solution, since it underestimates the energy contained in the intermediate frequencies. Close to the wall, viscosity will affect the flow more, such that the separation of the large and the small scales is smaller. Farther away from the wall, where the models are applied, viscosity is less important and a closeto-inertial range will appear (the straight line in the spectra indicates a $k^{-5 / 3}$ behavior). In this study, the standard approach of using the velocity in the logarithmic layer in order to infer the shear stress at the surface will be used, but it should be noted that there is room for further improvement. When comparing the different models in
Fig. 1, it is clear that the SG model is underestimating the fluctuations over all frequencies, whereas the MKP model and the proposed model are fairly similar, with good performance at the low and high frequencies. It is also clear that the slightly higher variance predicted by the MKP model is mainly due to a small overestimation of the low frequencies. In general the performance of the proposed model and the MKP model are on par and outstanding compared to the SG model.

\section{b. Numerical simulations}

A suite of large-eddy simulations of a neutrally stratified ABL was performed using the three different wall shear stress models (SG model, MKP model, and the proposed model), together with multiple numerical resolutions. The aim was to compare the different models to each other, but also to improve the understanding of wall boundary conditions and their effect on LES of atmospheric flows. For this, the filtered 

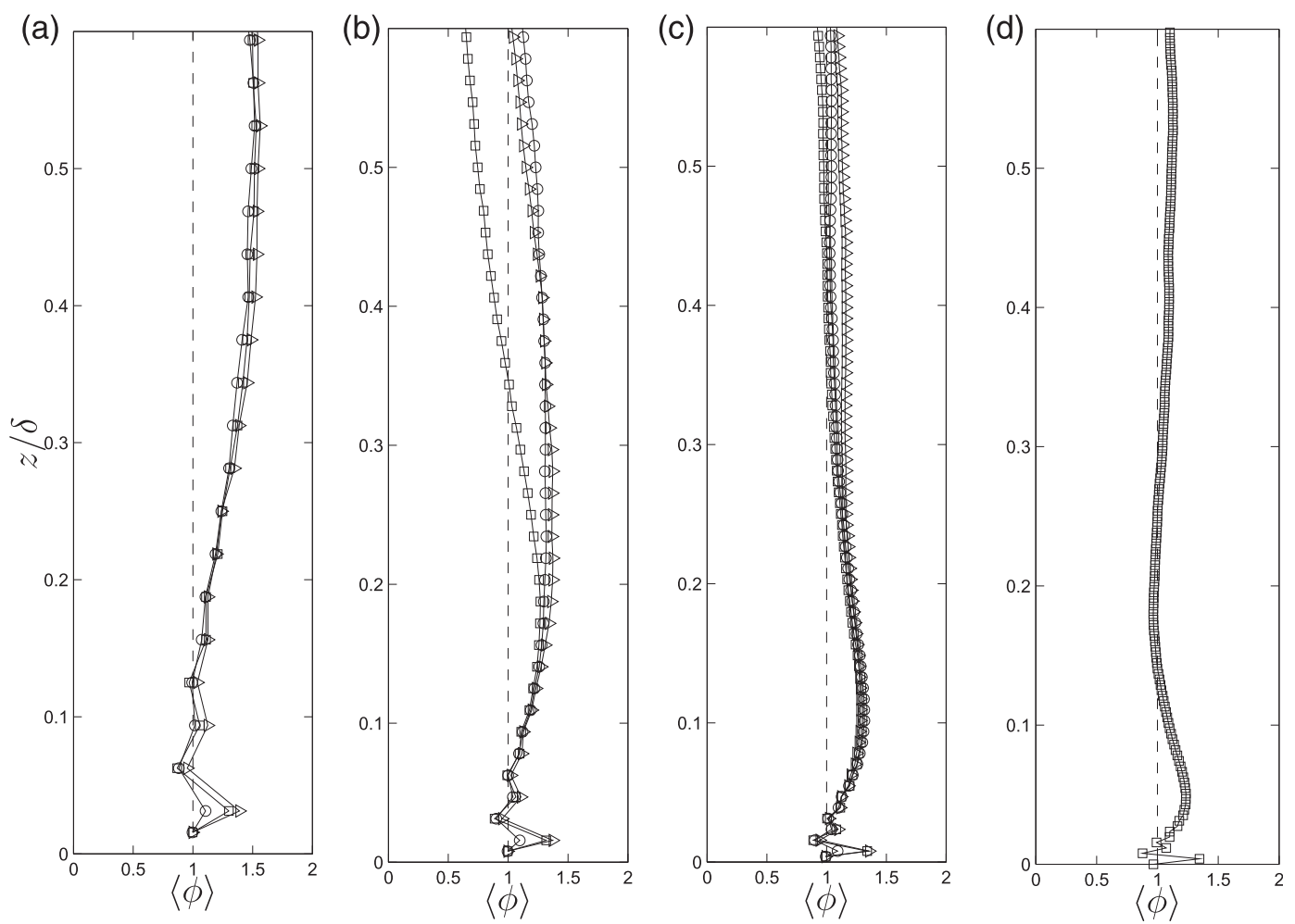

FIG. 3. Vertical profiles of the mean streamwise vertical derivative for the three different study models. The SG model is represented with open circles, the proposed model is represented with open squares, and the MKP model is represented with open triangles. The numerical resolutions (a) $32^{3}$, (b) $64^{3}$, (c) $128^{3}$, and (d) $256^{3}$ are presented.

nondimensional Navier-Stokes equations for an incompressible flow were numerically solved, namely,

$$
\begin{gathered}
\partial_{i} \tilde{u}_{i}=0 \\
\partial_{t} \tilde{u}_{i}+\partial_{j}\left(\tilde{u}_{i} \tilde{u}_{j}\right)=-\partial_{i} \tilde{p}^{*}-\delta_{i 1} \partial_{1} p_{\infty} / \rho-\partial_{j} \tau_{i j} .
\end{gathered}
$$

The flow was forced by a constant pressure gradient in the streamwise direction, which is included in the first term on the right-hand side, where $\tilde{p}^{*}$ is the filtered, modified pressure, normalized by the density, such as $\tilde{p}^{*}=\tilde{p} / \rho-\delta_{i 1} p_{\infty} / \rho+\tau_{k k} / 3$. Therefore, $\tau_{i j}$ is the trace-free subgrid shear stress (SGS), which was modeled using the Lagrangian scale-dependent model, as introduced by Bou-Zeid et al. (2005). Since the present study focuses on high Reynolds number and atmospheric flows, the first grid point was placed in the inertial region and viscous effects were neglected throughout the domain. A free-shear condition together with a zero vertical velocity was imposed as the top boundary conditions, and a staggered grid was used to numerically discretize the equations. For the bottom boundary condition, the different wall shear stress models being compared were imposed, with a constant and homogeneous surface roughness of $z_{0} / \delta=1 \times 10^{-4}$, where $\delta \sim 1 \mathrm{~km}$ is the height of the ABL, which is the characteristic length used to normalize the results. Following Moeng (1984), Albertson and Parlange (1999b), and Porté-Agel et al. (2000), a pseudospectral discretization was used for the horizontal directions and second-order finite differences were used in the vertical direction. The time integration was done with a fourth-order AdamsBashforth scheme, and the numerical code was parallelized using Message Passing Interface (MPI) for time optimization.

In total, 10 different $\mathrm{ABL}$ large-eddy simulations were performed using the SG model, the MKP model, and the proposed model. Three different grid resolutions were tested for each model $(32 \times 32 \times 32,64 \times 64 \times$ 64 , and $128 \times 128 \times 128$, corresponding to the $x, y, z$ directions, respectively). An additional high-resolution configuration of $256^{3}$ was also tested for the proposed model. The physical domain had a normalized size of $2 \pi \times 2 \pi \times 1$, and all simulations were allowed to run for an initial warm-up period of 27 nondimensional time units $t^{*}$ and the presented results were averaged over the following $9 t^{*}$, where $t^{*}=t \sqrt{\left\langle\tau_{w}\right\rangle} / \delta$ and $t$ is the dimensional time. 

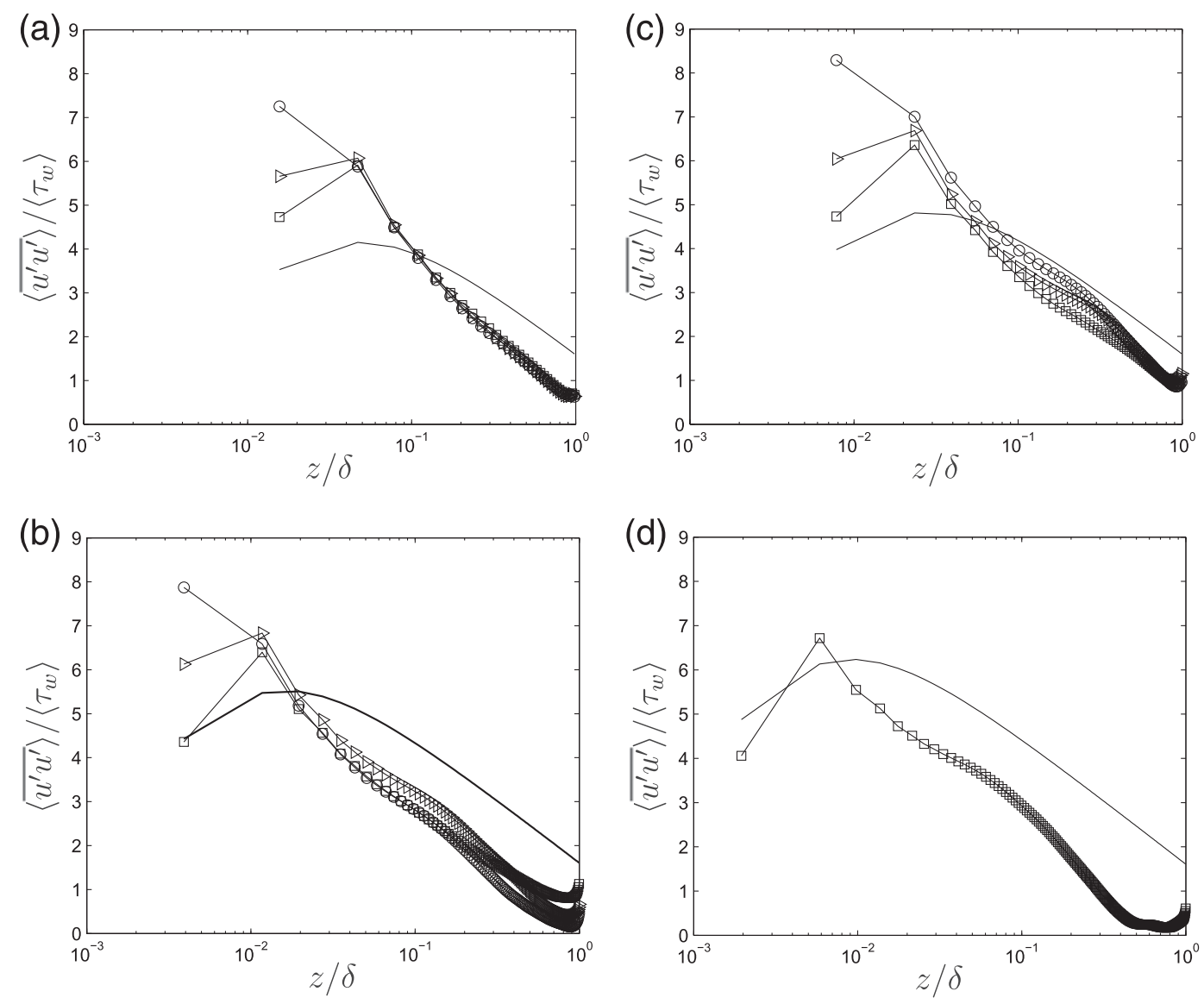

FIG. 4. Vertical profiles of the mean streamwise variance. The numerical resolutions (a) $32^{3}$, (b) $64^{3}$, (c) $128^{3}$, and (d) $256^{3}$ are presented. The SG model is represented with open circles, the proposed model is represented with open squares, and the MKP model is represented with open triangles.

Figure 2 shows vertical profiles of the mean streamwise velocity normalized by $\sqrt{\left\langle\tau_{w}\right\rangle}$. Figures $2 \mathrm{a}-\mathrm{d}$ show the cases with $32^{3}, 64^{3}, 128^{3}$, and $256^{3}$ numerical grid resolution, respectively. The numerical data agree well with the logarithmic profile for the first $12 \%-15 \%$ of the boundary layer thickness, which corresponds to the expected region for a logarithmic behavior. It is clear that all tested models return the logarithmic behavior and the differences are small. For the SG model and the MKP model this is expected, since they require the average value, based on the logarithmic behavior, as an input. However, the proposed model matches the logarithmic law equally well, even if computed only with local variables. Figure 3 shows the mean streamwise vertical derivative for the three different studied models. It can be observed that the vertical derivative for the SG model has small oscillations over the first $10 \%-15 \%$ and it approaches very closely the expected theoretical behavior of $\langle\phi\rangle=1$, when properly normalized. The other two models, the proposed model and the MKP model, have larger oscillations and perform worse than the SG model, presenting larger departures from the expected behavior. For a very thorough investigation of the gradient and the oscillations within them, see Brasseur and Wei (2010).

According to Hultmark et al. (2012), and as reflected through Eq. (10), a logarithmic profile is expected also for the variances, in the same region where the logarithmic profile is expected for the mean velocity $(z / H<$ $0.15)$. However, because of the numerical filtering inherent in LES, we cannot expect the resulting fluctuations to follow this behavior. Instead, we expect the variance to take a lower value than what Eq. (10) predicts, especially close to the wall. The correction method for this filtering [Eq. (13)] should capture the main features of the deviation from the logarithmic behavior. Therefore, the logarithmic behavior together with the correction scheme gives an analytical expression for the filtered variance, written as 

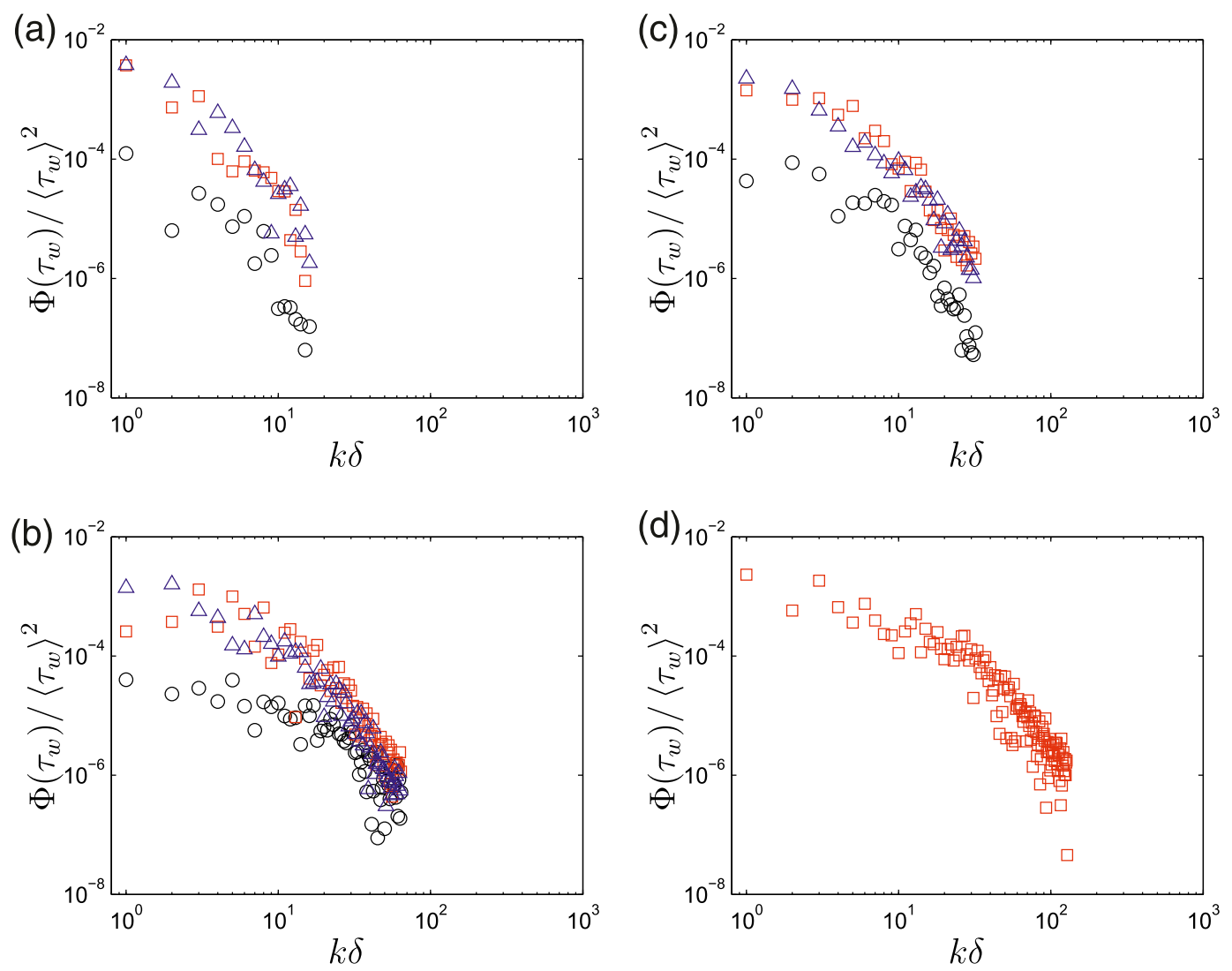

FIG. 5. Wavenumber spectra of the modeled wall shear stresses are presented for the numerical resolutions (a) $32^{3}$, (b) $64^{3}$, (c) $128^{3}$, and (d) $256^{3}$. Black open circles represent the SG model, red open squares represent the proposed model, and blue open triangles represent the MKP model.

$$
\left\langle\tilde{u}_{s}^{2}\right\rangle=\frac{1}{g(z / \delta)[1+0.1365(\Delta / z)]} .
$$

The numerical results can then be used to test the correction scheme itself, by comparing the variances of the numerically resolved velocity for the different resolutions to those as predicted by the correction scheme together with the logarithmic behavior. The solid line in Fig. 4 represents Eq. (22), and it is clear from the comparison between the numerical results and the analytical expression that the details of the numerical filtering are more complicated than what the correction suggests, but the main features are captured and the order of magnitude of the filtered energy is correct. Furthermore, the different wall shear stress models show different behaviors close to the wall. The variances, as computed with the proposed model and the MKP model, decrease close to the surface, in agreement with the corrected theoretical logarithmic profile, whereas the SG model does not. The $20 \%$ difference in fluctuating velocities can be explained by a difference in the transport of turbulent kinetic energy from the resolved scales toward the subgrid scales for the different models. Since the shear stress acts as the subgrid-scale model at the first grid point, the MKP model and the proposed model can be expected to behave similarly. The spectra of the resolved shear stress vary similarly for these two models. However, as shown in Fig. 1, the modeled shear stress from the SG model fluctuates much less, which would imply that less energy is dissipated and more is left in the resolved scales.

In Fig. 5 the spectra of the LES wall shear stress, for the different simulations, are presented. A time series of the enforced wall shear stress at the first grid point is continuously stored over the last $9 t^{*}$. The wavenumber spectra are then computed in the streamwise direction and ensemble averaged in the cross streamwise direction in space as well as in time. The corresponding spectra for all three models follow a similar shape, with the existence of a close-to-inertial range because the shear stress at the wall is computed using the velocity field away from the wall. However, similarly to what was seen in the experimental spectra (as seen in Fig. 1), there is a clear difference in magnitude between the SG model and the other 
two models, emphasizing the fact that the SG model tends to underestimate the fluctuations over all wavenumbers. Furthermore, the proposed model and the MKP model perform very similarly when numerically applied, which is supportive for the proposed model, since the MKP model was designed to reproduce the correct spectrum.

\section{Conclusions}

A new wall shear stress model is proposed that is based entirely on local quantities. By incorporating the results of recent high Reynolds number data regarding the streamwise turbulent fluctuations, a correction is formulated so that the model returns the correct average shear stress, yet allowing the shear stress to fluctuate in time and space. The proposed model was tested and its performance was compared to previous models, both on experimental data and on LES data. The first and second moments of the resulting shear stress as well as spectra were compared and evaluated. The new model was shown to perform equally well or better than previous, filtered, models, without the need for any kind of filter. A local model, such as the proposed one, is an important first step toward a wall shear stress model that can be applied to simulations of variable surfaces. This should allow LES to give more realistic simulations of the atmospheric boundary layer over complex terrain.

Acknowledgments. The authors thank Professor Charles Meneveau for his many useful hints and discussions. This work was made possible by support received through the Swiss National Science Foundation (Projects 200021 134892/1 and 200020125092$)$ and the Swiss National Supercomputing Centre (CSCS).

\section{REFERENCES}

Albertson, J. D., and M. B. Parlange, 1999a: Natural integration of scalar fluxes from complex terrain. Adv. Water Resour., 23, 239-252.

— plications for land-atmosphere interaction over complex terrain. Water Resour. Res., 35, 2121-2132.

Alfredsson, P. H., A. V. Johansson, J. H. Haritonidis, and H. Eckelmann, 1988: The fluctuating wall-shear stress and the velocity field in the viscous sublayer. Phys. Fluids, 31, 10261033.

Avissar, R., E. W. Eloranta, K. Gürer, and G. J. Tripoli, 1998: An evaluation of the large-eddy simulation option of the regional atmospheric modeling system in simulating a convective boundary layer: A FIFE case study. J. Atmos. Sci., 55, 1109-1130.

Bailey, S. C. C., and Coauthors, 2010: Turbulence measurements using a nanoscale thermal anemometry probe. J. Fluid Mech., 663, 160-179.

Belcher, S. E., I. N. Harman, and J. J. Finnigan, 2012: The wind in the willows: Flows in forest canopies in complex terrain. Annu. Rev. Fluid Mech., 44, 479-504, doi:10.1146/ annurev-fluid-120710-101036.

Bou-Zeid, E., C. Meneveau, and M. B. Parlange, 2004: Large-eddy simulation of neutral atmospheric boundary layer flow over heterogeneous surfaces: Blending height and effective surface roughness. Water Resour. Res., 40, W02505, doi:10.1029/ 2003WR002475.

$\longrightarrow, \ldots$, and ——, 2005: A scale-dependent Lagrangian dynamic model for large eddy simulation of complex turbulent flows. Phys. Fluids, 17, 025105, doi:10.1063/1.1839152.

- M. Parlange, and C. Meneveau, 2007: On the parametrization of surface roughness at regional scales. J. Atmos. Sci., 64, 216227.

Brasseur, J. G., and T. Wei, 2010: Designing large-eddy simulation of the turbulent boundary layer to capture law-of-the-wall scaling. Phys. Fluids, 22, 021303, doi:10.1063/1.3319073.

Chew, Y. T., B. C. Khoo, C. P. Lim, and C. J. Theo, 1998: Dynamic response of a hot-wire anemometer. Part II: A flush-mounted hot-wire and hot-film probes for wall shear stress measurements. Meas. Sci. Technol., 9, 765-778.

Gong, W., P. Taylor, and A. Dörnbrack, 1996: Turbulent boundarylayer flow over fixed aerodynamically rough two-dimensional sinusoidal waves. J. Fluid Mech., 312, 1-37.

Grötzbach, G., 1987: Direct numerical and large eddy simulations of turbulent channel flows. Complex Flow Phenomena and Modeling, N. P. Cheremisinoff, Ed., Vol. 6, Encyclopedia of Fluid Mechanics, Gulf Publishing, 1337-1391.

Hobson, J., N. Wood, and A. Brown, 1999: Large-eddy simulations of neutrally stratified flow over surfaces with spatially varying roughness length. Quart. J. Roy. Meteor. Soc., 125, 19371958.

Hultmark, M., M. Vallikivi, S. C. C. Bailey, and A. J. Smits, 2012: Turbulent pipe flow at extreme Reynolds numbers. Phys. Rev. Lett., 108, 094501, doi:10.1103/PhysRevLett.108.094501.

$\ldots, \ldots, \ldots$, and —_, 2013: Logarithmic scaling of turbulence in smooth and rough-walled pipe flow. J. Fluid Mech., 728, 376-395.

Khoo, B. C., Y. T. Chew, C. P. Lim, and C. J. Teo, 1998: Dynamic response of a hot-wire anemometer. Part I: A marginally elevated hot-wire probe for near-wall velocity measurements. Meas. Sci. Technol., 9, 751-763.

Landau, L., and E. Lifshitz, 1959: Fluid Mechanics. Pergamon Press, 536 pp.

Marusic, I., and W. D. C. Heuer, 2007: Reynolds number invariance of the structure inclination angle in wall turbulence. Phys. Rev. Lett., 99, 114504, doi:10.1103/PhysRevLett.99.114504.

_ G. J. Kunkel, and F. Porté-Agel, 2001: Experimental study of wall boundary conditions for large-eddy simulation. J. Fluid Mech., 446, 309-320.

Millikan, C. B., 1939: A critical discussion of turbulent flows in channels and circular tubes. Proceedings of the Fifth International Congress on Applied Mechanics, J. P. Den Hartog and H. Peters, Eds., Wiley, 386-932.

Moeng, C.-H., 1984: A large-eddy-simulation model for the study of planetary boundary-layer turbulence. J. Atmos. Sci., 41, 20522062.

Monin, A., and A. Obukhov, 1954: Basic laws of turbulence mixing in the surface layer of the atmosphere. Tr. Geofiz. Inst., Akad. Nauk SSSR, 24, 163-187.

Piomelli, U., J. Ferziger, P. Moin, and J. Kim, 1989: New approximate boundary conditions for large eddy simulations of wall-bounded flows. Phys. Fluids, 1A, 1061, doi:10.1063/ 1.857397 . 
Porté-Agel, F., C. Meneveau, and M. B. Parlange, 2000: A scaledependent dynamic model for large-eddy simulation: Application to a neutral atmospheric boundary layer. J. Fluid Mech., 415, 261-284.

—, M. Pahlow, C. Meneveau, and M. B. Parlange, 2001: Atmospheric stability effect on subgrid-scale physics for large-eddy simulation. Adv. Water Resour., 24, 1085-1102.

Prandtl, L., 1925: Bericht über Untersuchungen zur ausgebildeten Turbulenz. Z. Angew. Math. Mech., 5, 136-139.

— 1932: Zur turbulenten Strömung in Röhren und längs Platten. Ergeb. Aerodyn. Versuchsanst., 4, 18-29.

Schumann, U., 1975: Subgrid scale model for finite difference simulations of turbulent flows in plane channels and annuli. J. Comput. Phys., 18, 376-404.

1990: Large-eddy simulation of the up-slope boundary layer. Quart. J. Roy. Meteor. Soc., 116, 637-670.
Shaw, R., and U. Schumann, 1992: Large-eddy simulation of turbulent flow above and within a forest. Bound.-Layer Meteor., 61, 47-64.

Smits, A. J., J. Monty, M. Hultmark, S. C. C. Bailey, M. Hutchins, and I. Marusic, 2011: Spatial resolution correction for turbulence measurements. J. Fluid Mech., 676, 41-53.

Stoll, R., and F. Porté-Agel, 2006: Effect of roughness on surface boundary conditions for large-eddy simulation. Bound.-Layer Meteor., 118, 169-187.

Tennekes, H., and J. L. Lumley, 1972: A First Course in Turbulence. MIT Press, $300 \mathrm{pp}$.

Townsend, A. A., 1976: The Structure of Turbulent Shear Flow. 2nd ed. Cambridge University Press, 429 pp.

Vallikivi, M., M. Hultmark, S. C. C. Bailey, and A. J. Smits, 2011: Turbulence measurements in pipe flow using a nano-scale thermal anemometry probe. Exp. Fluids, 51, 1521-1527. 